\title{
Double Penetrated Duodenal Wall during Endoscopic Ultrasound-Guided Choledochoduodenostomy
}

\author{
Hiroshi Kawakami*, Masaki Kuwatani ${ }^{\dagger}$, and Naoya Sakamoto ${ }^{\ddagger}$
}

${ }^{*}$ Department of Gastroenterology and Hepatology, ${ }^{\dagger}$ Division of Endoscopy, Hokkaido University Hospital, and ${ }^{\ddagger}$ Department of Gastroenterology and Hepatology, Hokkaido University Graduate School of Medicine, Sapporo, Japan

Endoscopic ultrasound-guided choledochoduodenostomy (EUS-CDS) using a covered self-expandable metallic stent (CSEMS) was developed as an alternative biliary drainage technique in patients with failed endoscopic retrograde cholangiopancreatography (ERCP). ${ }^{1,2}$ Here, we present a case that underwent EUS-CDS with a partial CSEMS, resulting in a doubly penetrated duodenal wall for malignant distal bile duct obstruction.

A 44-year-old woman with cancer of unknown origins and obstructive jaundice was referred to our hospital. We performed ERCP, but selective bile duct cannulation was unsuccessful even with precutting. EUS-CDS was attempted using a curved-linear echoendoscope. Following the puncture of the common bile

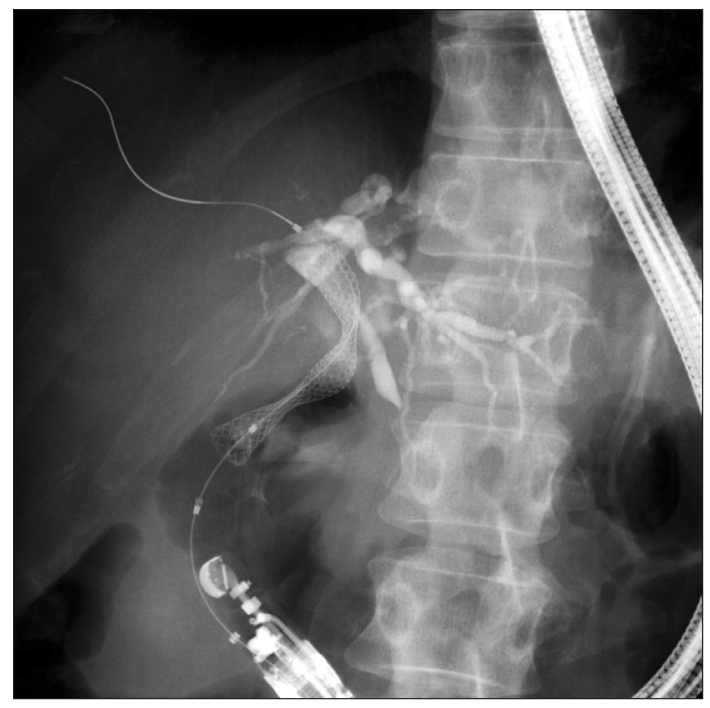

Fig. 1. Radiograph showing a candy-like appearance during endoscopic ultrasound-guided choledochoduodenostomy. duct from the duodenal bulb using a 19-gauge needle (EchoTip ${ }^{\circledR}$ Ultra; Cook Japan, Tokyo, Japan), a 0.025-inch guidewire (VisiGlide $^{\mathrm{TM}}$; Olympus Medical Systems Corp., Tokyo, Japan) was inserted into the intrahepatic bile duct. The needle tract was dilated by a 6-F wire-guided diathermic dilator (Cysto-Gastro-Set; Endo-Flex GmbH, Voerde, Germany) using a blended cut mode. After the dilation of the tract, we inserted a partial CSEMS through the fistula tract under fluoroscopic guidance and direct visualization. The EUS-CDS procedure was performed quickly; however, the fluoroscopic view revealed partial CSEMS showing a candy-like appearance (Fig. 1). At first, we thought the candylike appearance was caused by the long distance between the bile duct and the duodenal wall. ${ }^{3}$ However, no dislocation was

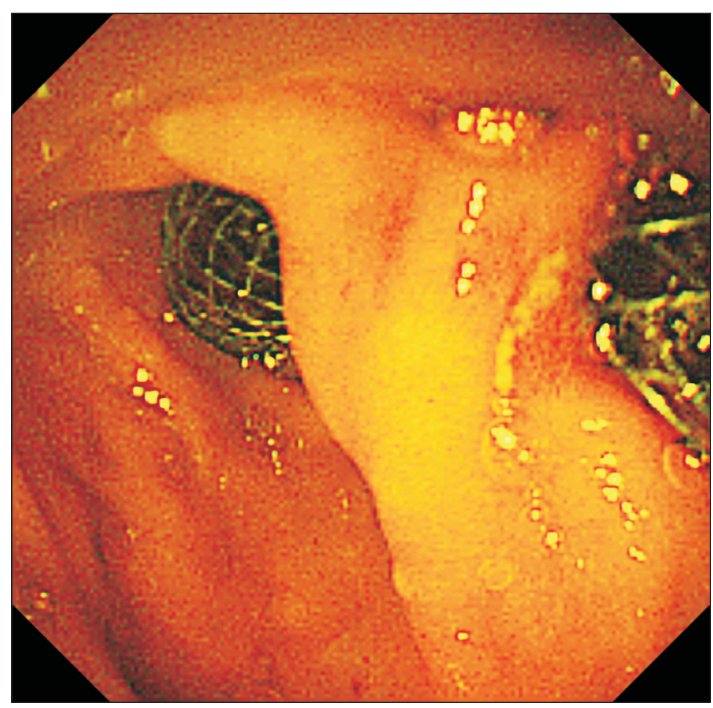

Fig. 2. Endoscopic image immediately after endoscopic ultrasoundguided choledochoduodenostomy showing the doubly penetrated duodenal wall at the duodenal bulb.

Correspondence to: Hiroshi Kawakami

Department of Gastroenterology and Hepatology, Hokkaido University Hospital, North 14, West 5, Kita-ku, Sapporo 060-8648, Japan

Tel: +81-11-716-1161, Fax: +81-11-706-7867, E-mail: hiropon@med.hokudai.ac.jp

Received on May 27, 2015. Revised on June 15, 2015. Accepted on June 16, 2015. Published online October 19, 2015

pISSN 1976-2283 eISSN 2005-1212 http://dx.doi.org/10.5009/gnl15238

() This is an Open Access article distributed under the terms of the Creative Commons Attribution Non-Commercial License (http://creativecommons.org/licenses/by-nc/4.0) which permits unrestricted non-commercial use, distribution, and reproduction in any medium, provided the original work is properly cited. 
observed after releasing the partial CSEMS, which indicated that no distal migration occurred at the puncture site. Eventually, we confirmed that the candy-like appearance resulted from the doubly penetrated duodenal wall (Fig. 2). Fortunately, no additional interventions were required.

Recently, EUS-CDS using CSEMS has been reported and is favored for reducing bile leakage and achieving prolonged stent patency. ${ }^{2}$ Forward-viewing echoendoscopes may be useful to prevent doubly penetrating the duodenal wall with a needle compared with curved-linear ones for the following differences: the location of the echo probe and its short length. The endoscopist should check the endoscopic view during CSEMS deployment at the anastomotic site. However, doubly penetrating the duodenal wall during EUS-CDS cannot be avoided at that time. The findings described here suggest that more attention should be paid to the possibility of doubly penetrating the duodenal wall when performing EUS-CDS using curved-linear echoendoscopes.

\section{CONFLICTS OF INTEREST}

No potential conflict of interest relevant to this article was reported.

\section{REFERENCES}

1. Itoi $\mathrm{T}$, Isayama $\mathrm{H}$, Sofuni $\mathrm{A}$, et al. Stent selection and tips on placement technique of EUS-guided biliary drainage: transduodenal and transgastric stenting. J Hepatobiliary Pancreat Sci 2011;18:664-672.

2. Kawakubo K, Isayama H, Kato H, et al. Multicenter retrospective study of endoscopic ultrasound-guided biliary drainage for malignant biliary obstruction in Japan. J Hepatobiliary Pancreat Sci 2014;21:328-334.

3. Kawakami H, Kuwatani M, Kawakubo K, et al. Candy-like sign during endoscopic ultrasound-guided choledochoduodenostomy as an indication of the long distance between the bile duct and duodenal wall. Endoscopy 2014;46 Suppl 1 UCTN:E406-E407. 